\title{
Optimizing contrast agents with respect to reducing beam hardening in nonmedical X-ray computed tomography experiments
}

\author{
Yoshito Nakashima* and Tsukasa Nakano \\ National Institute of Advanced Industrial Science and Technology, Geological Survey of Japan, \\ Tsukuba, Ibaraki, Japan
}

Received 26 April 2013

Revised 17 October 2013

Accepted 1 November 2013

\begin{abstract}
Iodine is commonly used as a contrast agent in nonmedical science and engineering, for example, to visualize Darcy flow in porous geological media using X-ray computed tomography (CT). Undesirable beam hardening artifacts occur when a polychromatic X-ray source is used, which makes the quantitative analysis of CT images difficult. To optimize the chemistry of a contrast agent in terms of the beam hardening reduction, we performed computer simulations and generated synthetic CT images of a homogeneous cylindrical sand-pack (diameter, 28 or $56 \mathrm{~mm}$; porosity, 39 vol.\%) saturated with aqueous suspensions of heavy elements assuming the use of a polychromatic medical CT scanner. The degree of cupping derived from the beam hardening was assessed using the reconstructed CT images to find the chemistry of the suspension that induced the least cupping. The results showed that (i) the degree of cupping depended on the position of the $\mathrm{K}$ absorption edge of the heavy element relative to peak of the polychromatic incident X-ray spectrum, (ii) ${ }_{53}$ I was not an ideal contrast agent because it causes marked cupping, and (iii) a single element much heavier than ${ }_{53} \mathrm{I}\left({ }_{64} \mathrm{Gd}\right.$ to ${ }_{79} \mathrm{Au}$ ) reduced the cupping artifact significantly, and a four-heavy-element mixture of elements from ${ }_{64} \mathrm{Gd}$ to ${ }_{79} \mathrm{Au}$ reduced the artifact most significantly.
\end{abstract}

Keywords: Beam hardening, contrast agent, cupping, K absorption edge, lanthanoid, X-ray CT

\section{Introduction}

X-ray contrast agents are used to enhance differentiation among different phases in samples [1]. Iodine, with its large atomic number $(Z)$, is one of the most commonly used contrast agents in nonmedical science and engineering. For example, it is used in laboratory experiments by means of medical and microfocus X-ray computed tomography (CT) systems to help visualize the flow or diffusion of fluids in complex, porous geological media [2-5]. Humans and animals have a low density $\left(\approx 1-2 \mathrm{~g} / \mathrm{cm}^{3}\right)$; however, porous geological media generally have a much higher density $\left(\approx 2-4 \mathrm{~g} / \mathrm{cm}^{3}\right)$, which means that a pore fluid with an iodine concentration much larger than that needed for the CT measurements of humans and animals must be used (e.g., a KI solution of $0.6 \mathrm{~mol} / \mathrm{L}[2])$.

\footnotetext{
${ }^{*}$ Corresponding author: Yoshito Nakashima, National Institute of Advanced Industrial Science and Technology (AIST), Geological Survey of Japan, Central 7, Higashi 1-1-1, Tsukuba, Ibaraki 305-8567, Japan. Fax: +81 29861 3618; E-mail: nakashima.yoshito@aist.go.jp.
}

0895-3996/14/\$27.50 (c) 2014 - IOS Press and the authors. All rights reserved

This article is published online with Open Access and distributed under the terms of the Creative Commons Attribution NonCommercial License. 
High-concentration contrast agents enhance beam hardening when a polychromatic X-ray source is used. As the polychromatic X-ray beam passes through a sample, the X-ray spectrum becomes harder (i.e., its mean X-ray energy increases), because the lower-energy photons are absorbed more rapidly particularly by the contrast agent compared with the higher-energy photons. The obtained projection (i.e., logarithmic attenuation) is no longer a linear function of the sample thickness. This beam hardening yields the following artifacts in the reconstructed CT images [6-9]: cupping (a profile of the voxel values across a homogeneous sample displays a characteristic cupped shape); dark streaks between two objects having strong X-ray attenuation coefficients. The beam hardening artifact is useful for limited applications $[10,11]$. In most cases, however, the artifact is undesirable because the spatial distribution of the voxel values in a homogeneous sample is distorted to be heterogeneous. Example includes CT image-based calculations of the relative permeability curve and residual oil saturation [12] by means of the partial-volume effect [13-15] in two-phase Darcy flow experiments [3,4,15].

Conventional techniques to reduce beam hardening include modifying the $\mathrm{X}$-ray projection data or reconstructed images [8,16-19], or using a metal filter [20,21] or dual-energy X-ray source [9,22]. However, these techniques have some critical drawbacks. Modification of the projection data or reconstructed images distorts the voxel values of CT images, which is undesirable in material identification studies [10, 23,24]. Furthermore, some modification methods assume that the sample is homogeneous and/or that data on the density and chemistry of the sample are readily available, which is not always true for heterogeneous or complex geological samples; other methods require complicated digital processing of the projection data and images that takes a considerable amount of CPU time. The use of metal filters reduces X-ray intensity and increases random noise in the reconstructed images. Dual-energy X-ray CT is time-consuming compared with single-energy CT, and its use is not appropriate to the monitoring of rapidly changing phenomena within samples; it also reduces the potential lifetime of the expensive $\mathrm{X}$-ray tube and detector.

An alternative to the methods mentioned above is to choose a contrast agent with a specific atomic number [25,26]. Nakashima [27] found that an aqueous solution of ${ }_{74} \mathrm{~W}$ significantly reduces beam hardening in geotechnical CT experiments compared with ${ }_{53} \mathrm{I}$, suggesting that elements heavier than iodine may be promising for nonmedical research. To examine the feasibility of this method, we performed a series of CT simulations. The simulations mimic the cupping artifact which often occurs in the CT images of homogeneous porous geological samples saturated with heavy element-bearing fluids [4, 27]. Assuming the use of a polychromatic medical CT scanner, synthetic CT images were reconstructed for porous sand-pack samples saturated with aqueous suspensions of various concentrations of different heavy elements. The degree of cupping derived from beam hardening in the synthetic CT images was quantitatively assessed to find the heavy-element suspension that induced the least cupping.

\section{CT simulations}

\subsection{Basic principles}

For a porous sample saturated with a contrast agent to be beam hardening-free, it should have a flat, or energy-independent, X-ray linear attenuation coefficient (LAC) spectrum. However, the LAC spectrum of a saturated geological sample with iodine is far from flat (Fig. 1, purple line), yielding considerable beam hardening. Contrast agents containing a heavy element whose $\mathrm{K}$ absorption edge is located near the peak of the polychromatic X-ray source spectrum of Fig. 1, induce less beam hardening because the abrupt increase in the LAC value at the K absorption edge flattens the LAC spectrum $[10,26]$. This 
suggests that a contrast agent better than iodine can be expected among the heavy elements whose $\mathrm{K}$ absorption edge is located closer to the peak of the polychromatic X-ray source spectrum. Furthermore, a selected mixture of heavy elements with different $\mathrm{K}$ absorption edges may yield an even flatter LAC spectrum (Fig. 1, red and green lines) compared with a contrast agent containing a single heavy element.

Here, we used computer simulation to search for the contrast agent that induces the least beam hardening (cupping). We prepared a range of virtual contrast agents by mixing heavy elements in different weight fractions and suspending them in bulk water, and injected them into virtual porous geological samples. This process mimics the sample preparation for the laboratory CT experiments of Darcy flow in porous geological samples $[4,15,27]$. Two-dimensional (2-D) CT images of the porous geological samples saturated with the various contrast agents were reconstructed assuming the use of a polychromatic medical CT scanner. Line profiles of the voxel value were collected, and the degree of cupping artifact in the homogeneous sample images was quantitatively analyzed. Through trial and error, we determined the chemical composition of the best contrast agent for the virtual sample examined. The best contrast agent was considered the heavy element-bearing aqueous suspension that induced the least cupping artifact in the line profile analysis of the CT images. Note that we did not directly pursue the geometrical flatness of the LAC spectrum of Fig. 1 because sample size, which is an important factor affecting cupping, is not accounted for in the LAC spectrum.

\subsection{Simulations}

Toyoura sand from Yamaguchi, Japan, which is a common standard sand used in geotechnical research $[27,28]$, was chosen as the virtual geo-material for this study (grain density, $2.65 \mathrm{~g} / \mathrm{cm}^{3}$; chemical composition, $\mathrm{SiO}_{2}, 92.6 ; \mathrm{Al}_{2} \mathrm{O}_{3}, 3.7 ; \mathrm{Fe}_{2} \mathrm{O}_{3}, 0.7 ; \mathrm{CaO}, 0.5 ; \mathrm{MgO}, 0.2 ;$ loss on ignition, 0.5 wt.\% (total, $98.2 \mathrm{wt} . \%)$ ). The sand was packed in a cylindrical tube of negligible thickness to produce a sand pack with a porosity of 39 vol. $\%$. Two diameters of sand pack ( 90 voxel $=28 \mathrm{~mm}$ and 180 voxel $=56 \mathrm{~mm})$ were used to examine how sample size affects cupping. The grain diameter of Toyoura sand is about $0.2 \mathrm{~mm}$ [28], which is significantly smaller than the typical CT-slice thickness of a few millimeters. Thus, we assumed that the individual pore spaces and sand grains were indistinguishable and the sand pack was homogeneous due to the partial-volume effect [13-15], and that the voxel-value distribution within the synthetic CT image of the sand pack would be homogeneous if the cupping artifact and filterderived Gibbs phenomenon were both negligible. It should be noted that our simulations are not for the segmentation of solid grains and fluid-filled pores using pore- or grain-scale images [8].

The virtual Toyoura sand-packs were completely saturated with an aqueous suspension containing one or more heavy elements. The heavy elements were dispersed in pure water as fine particles $[29,30]$ much smaller than the voxel size of the synthetic CT image, which again yielded a homogeneous voxel-value distribution in the $\mathrm{CT}$ image if both the beam hardening (cupping) and filter-derived Gibbs phenomenon were negligible.

According to Nakashima and Nakano [10], a heavy element induces less cupping the closer the energy of the element's $\mathrm{K}$ absorption edge is to the peak of the polychromatic spectrum of the X-ray source. Ten heavy elements used in medical and life sciences [29-35] were chosen as candidate contrast agents; ${ }_{53} \mathrm{I},{ }_{58} \mathrm{Ce},{ }_{60} \mathrm{Nd},{ }_{64} \mathrm{Gd},{ }_{67} \mathrm{Ho},{ }_{70} \mathrm{Yb},{ }_{72} \mathrm{Hf},{ }_{74} \mathrm{~W},{ }_{79} \mathrm{Au}$, and ${ }_{83} \mathrm{Bi}$. The $\mathrm{K}$ absorption edges of the ten heavy elements range from $33.2 \mathrm{keV}\left({ }_{53} \mathrm{I}\right)$ to $90.5 \mathrm{keV}\left({ }_{83} \mathrm{Bi}\right)$, which covers almost the whole polychromatic $\mathrm{X}$-ray photon energy-range of a medical CT scanner for two acceleration voltages (Fig. 1). The number of heavy elements was limited to 10 to keep the CPU time required for the simulations realistic. For each heavy element suspension, the concentration range was from 0 to $8 \mathrm{wt} . \%$ (in increments of $1 \mathrm{wt} . \%$ ), with 

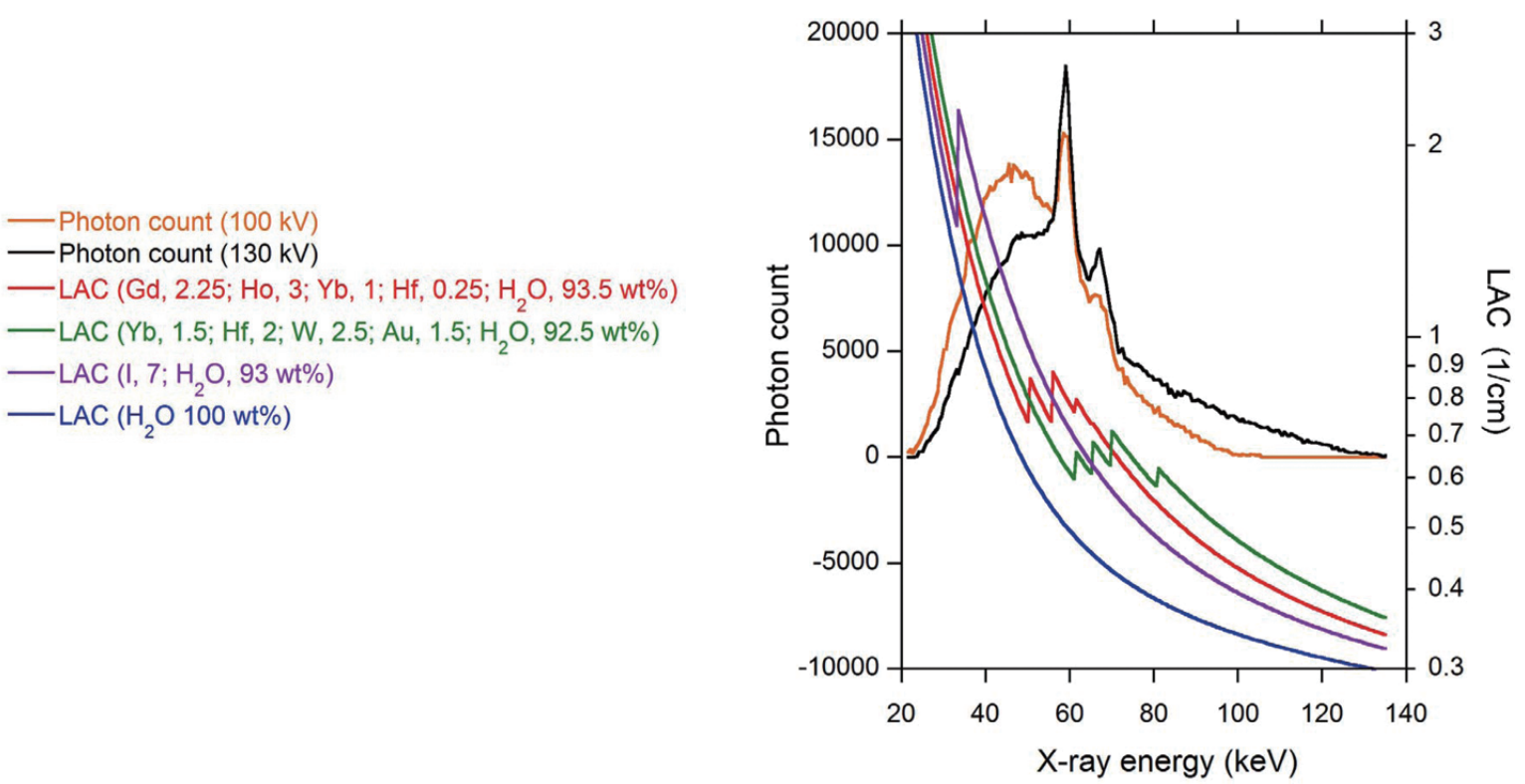

Fig. 1. Photon energy histogram of the primary X-rays emitted from an X-ray tube at acceleration voltages of 100 or $130 \mathrm{kV}$ [39]. Energy-dependent LAC spectra for fluid-saturated Toyoura sand-packs (porosity, 39 vol.\%) calculated by using the XCOM database [36] are superimposed. The four kinds of pore fluid chemistry are shown. Line profiles of the simulated CT images corresponding to the three kinds of the fluid chemistry (i.e., except for the case of $\mathrm{H}_{2} \mathrm{O} 100$ wt.\%) are shown in Fig. 3.

the further constraint that the maximum total heavy elements in the aqueous suspensions containing a mixture of heavy elements was $8 \mathrm{wt}$ \%, except for a pedagogical case shown in Fig. 2b. With these conditions, the various heavy elements were virtually mixed and suspended in water, and then injected into the simulated porous Toyoura sand-pack. As a result, the overall chemical composition (in wt.\%) of the porous sand pack saturated with aqueous suspension containing heavy elements, essential to the CT image synthesis, was fixed.

The chemical composition data (in wt.\%) of the whole sample were transferred to the XCOM database [36] to output the energy-dependent X-ray mass attenuation coefficients (MAC). The MAC spectrum can be converted into a LAC spectrum by multiplying the bulk density value of the sand-pack sample saturated with an aqueous suspension containing heavy elements. The bulk density of the sample was calculated as follows. We assumed that the fine particles of the heavy elements are made of a simple substance that possesses the solid grain density values available in the literature (e.g., $7.90 \mathrm{~g} / \mathrm{cm}^{3}$ for gadolinium, $19.3 \mathrm{~g} / \mathrm{cm}^{3}$ for tungsten, see [37]). The saturated sand pack sample was assumed to be a mechanical mixture of water, sand grains, and the fine powder of the simple substance. It is then possible to calculate the bulk density of the mixture using the data on the density and weight fraction of each component $[10,38]$. The powder-dispersed suspension model used in the present study may not exactly be valid for iodine and the lanthanoids because for these elements electrolytes (not insoluble fine powders) are often used as contrast agents. However, we have confirmed that this powder-dispersed suspension model reproduced well the experimentally measured bulk density values for dilute aqueous solutions of electrolytes (e.g., $\mathrm{KI}$ and $\mathrm{CeCl}_{3}$, see [10]).

By using a personal computer (Intel Core i7 3970X CPU (3.50 GHz) running Windows $\left.7{ }^{\circledR}\right)$, we simulated 2-D CT imaging of the cylindrical homogeneous sand pack having the LAC spectrum calculated 

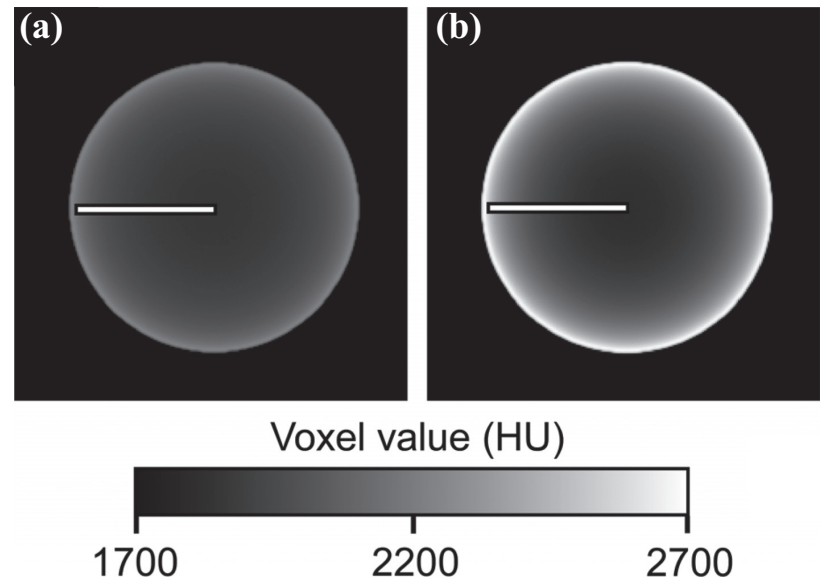

Fig. 2. Example 2-D CT images of a simulated Toyoura sand-pack saturated with an aqueous heavy-element suspension (porosity, 39 vol.\%; diameter, $56 \mathrm{~mm}$; acceleration voltage, $130 \mathrm{kV}$ ). Image dimension, $240^{2}$ voxels $=75^{2} \mathrm{~mm}^{2}$. (a) One of the best contrast agents that induced the least cupping ( $\mathrm{Yb}, 1.5 ; \mathrm{Hf}, 2.0 ; \mathrm{W}, 2.5 ; \mathrm{Au}, 1.5 ; \mathrm{H}_{2} \mathrm{O}, 92.5$ wt.\%; see D2 in Table 1). (b) An aqueous suspension of iodine (I, 10; $\mathrm{H}_{2} \mathrm{O}, 90$ wt.\%) exhibiting marked cupping. Line profiles along the horizontal baselines (85-voxels long) are shown in Fig. 3. (Colours are visible in the online version of the article; http://dx.doi.org/10.3233/XST-130411)

by the above mentioned method. The details of this CT simulation method have been described previously $[10,18]$. Briefly, we assumed the use of a medical X-ray CT scanner that has the polychromatic $\mathrm{X}$-ray source spectrum shown in Fig. 1 (acceleration voltage, $100 \mathrm{kV}$ or $130 \mathrm{kV}$ [39]). The projection data acquisition system consists of a pencil beam and a single detector. The calculated LAC spectrum was combined with the photon energy distribution of the pencil beam shown in Fig. 1 to output a sinogram by using Beer's law. The number of projections was 805 per $180^{\circ}$ rotation. We used the sinogram to reconstruct a 2-D 16-bit CT image by using a convolution back projection algorithm with a Chesler filter. The field of view of the original reconstructed image was $160 \times 160 \mathrm{~mm}^{2}=512 \times 512$ voxels (in-plane voxel dimension, $160 / 512 \approx 0.31 \mathrm{~mm}$ ) according to the specification of the medical CT scanner [39]; the original image was trimmed to extract a region of interest $\left(240^{2}\right.$ voxels $=75^{2} \mathrm{~mm}^{2}$, see Fig. 2). We applied a line-profile analysis of the voxel values to the trimmed image exhibiting cupping to evaluate the effects of the chemical composition of the contrast agents on the degree of cupping artifact.

\section{Results}

Example reconstructed CT images of the sand pack saturated with a contrast agent are shown in Fig. 2 for a pedagogical purpose. The CPU time required to obtain a single CT image of Fig. 2 was about six seconds. The cupping derived from beam hardening is responsible for the inhomogeneous image showing that the voxels are dark in the sample center and bright near the sample rim. The difference between the two images in the figure demonstrates that the degree of cupping depends on the chemistry of the contrast agent.

Example effects of the pore fluid chemistry on the degree of cupping obtained by the line-profile analysis are shown in Fig. 3. The line-profile analysis was performed as follows: the one-dimensional distribution of the voxel values along a horizontal baseline (Fig. 2) crossing the CT image was sampled. The voxel value near the rim of the cylindrical sample image is distorted by the reconstruction filter (e.g., the Gibbs phenomenon) $[18,24]$. Thus, we discarded several voxels near the rim, and used two baselines: 
- 90 voxel, $100 \mathrm{kV}\left(\mathrm{I}, 7 ; \mathrm{H}_{2} \mathrm{O}, 93 \mathrm{wt} . \%\right)$

- 90 voxel, $100 \mathrm{kV}\left(\mathrm{Gd}, 6 ; \mathrm{H}_{2} \mathrm{O}, 94 \mathrm{wt} . \%\right)$

- 90 voxel, $100 \mathrm{kV}\left(\mathrm{Gd}, 2.25 ; \mathrm{Ho}, 3 ; \mathrm{Yb}, 1 ; \mathrm{Hf}, 0.25 ; \mathrm{H}_{2} \mathrm{O}, 93.5 \mathrm{wt} . \%\right)$

- 180 voxel, $130 \mathrm{kV}\left(\mathrm{I}, 10 ; \mathrm{H}_{2} \mathrm{O}, 90\right.$ wt.\%)

-180 voxel, $130 \mathrm{kV}\left(\mathrm{W}, 8 ; \mathrm{H}_{2} \mathrm{O}, 92 \mathrm{wt} . \%\right)$

- 180 voxel, $130 \mathrm{kV}$ (Yb, 1.5; Hf, 2; W, 2.5; Au, 1.5; $\left.\mathrm{H}_{2} \mathrm{O}, 92.5 \mathrm{wt} . \%\right)$

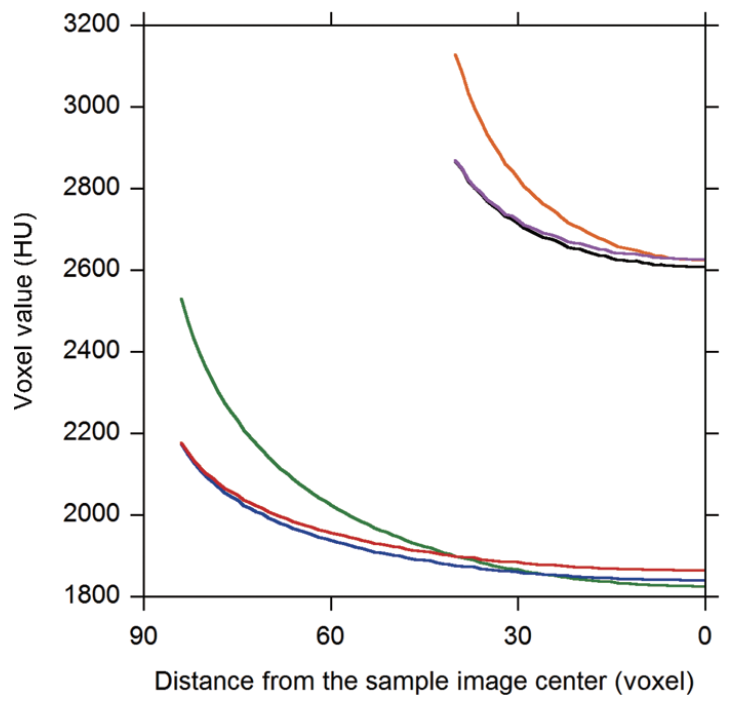

Fig. 3. Example effects of the pore fluid chemistry on the degree of cupping for a specified sand-pack diameter $(90$ voxel $=$ $28 \mathrm{~mm}$ and 180 voxel $=56 \mathrm{~mm}$ ) and acceleration voltage. Line profiles along the baselines (see Fig. 2) for six fluid-saturated Toyoura sand packs are shown.

one 85-voxels long (i.e., 27-mm long) for the sand pack with a diameter of 180 voxels (Fig. 2), and one 41-voxels long (i.e., 13-mm long) for the sand pack with a diameter of 90 voxels. The red and green line-profiles in Fig. 3 corresponding to the images of Figs 2(a), (b), respectively demonstrate the evident dependence of the degree of cupping on the chemistry of the contrast agent. Two parameters, $P_{\min }$ and $\Delta$, were defined and calculated using the line profile to quantitatively evaluate the degree of cupping: $P_{\min }$ is the minimum of the line profile (i.e., the voxel value at the sample image center); $\Delta$ is the difference between the maximum and minimum of the voxel values of the line profile. The parameter $\Delta$ is zero for a completely cupping-free CT image. In this study, the best contrast agent refers to the aqueous heavy-element suspension that resulted in the smallest $\Delta$ value for a specified $P_{\min }$ value.

The unit of the voxel values obtained by medical CT scanners is conventionally converted from $1 / \mathrm{cm}$ into Hounsfield unit (HU), which is normalized using the voxel value of a bulk water sample [3]. We generated CT images of cylindrical samples of pure bulk water (i.e., without sand and heavy elements). The obtained $\left(P_{\min }, \Delta\right)$ values for the acceleration voltages and sample diameters shown in Figs $4 \mathrm{a}$, $4 \mathrm{~b}, 4 \mathrm{c}$, and $4 \mathrm{~d}$ were $(0.235,0.0026),(0.216,0.0019),(0.231,0.0052)$, and $(0.214,0.0038)$, respectively (unit, $1 / \mathrm{cm}$ ). As these $\Delta$ values were small and the $P_{\min }$ values were almost identical, for simplicity, we used a single value of $0.2241 / \mathrm{cm}$, which is the arithmetic average of the four $P_{\min }$ values, to convert the voxel values from $1 / \mathrm{cm}$ into $\mathrm{HU}$ : voxel value $($ in $\mathrm{HU})=1000 \times($ voxel value $($ in $1 / \mathrm{cm})-0.224) / 0.224$. Hereafter, HU is used as the unit of the voxel value (not $1 / \mathrm{cm}$ ).

The results of the search for the best contrast agent are summarized in Figs 4 and 5. The number of data points (black circles), which represent a mixture of all 10 heavy elements (i.e., I, Ce, $\mathrm{Nd}, \mathrm{Gd}$, Ho, $\mathrm{Yb}, \mathrm{Hf}, \mathrm{W}, \mathrm{Au}$, and Bi) is 43,758 in Figs 4(a) to (d). The results obtained for aqueous suspensions of a single heavy element are superimposed on top (i.e., single-element suspensions of I, Gd, Ho, Yb, Hf, $\mathrm{W}, \mathrm{Au}$, and $\mathrm{Bi}$ ). Analysis of the results revealed that mixtures of only four heavy elements produced the smallest $\Delta$ value for almost all specified $P_{\min }$ values. Thus, we performed additional simulations for different mixtures of four heavy elements (Figs 4 and 5). In these mixtures, the concentration of each heavy element ranged from 0.25 to $8 \mathrm{wt} . \%$, and the maximum total heavy elements in the aqueous 


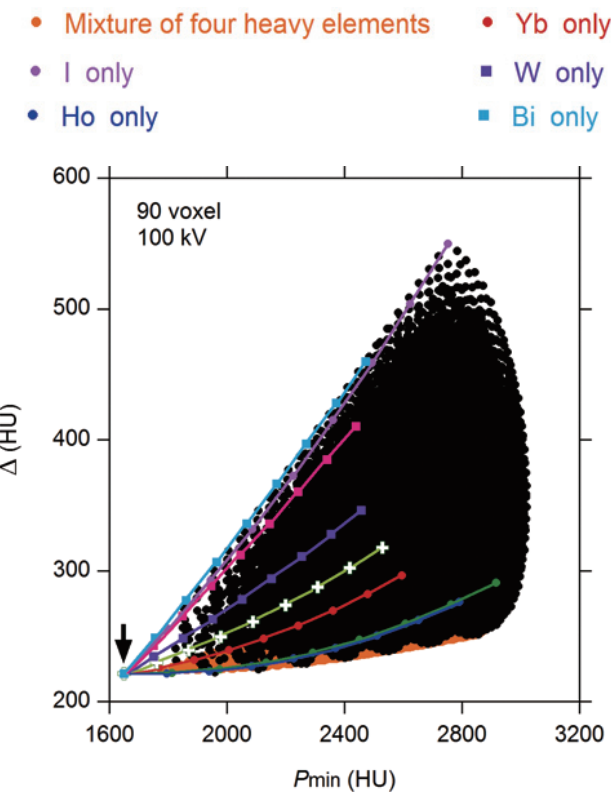

(a)

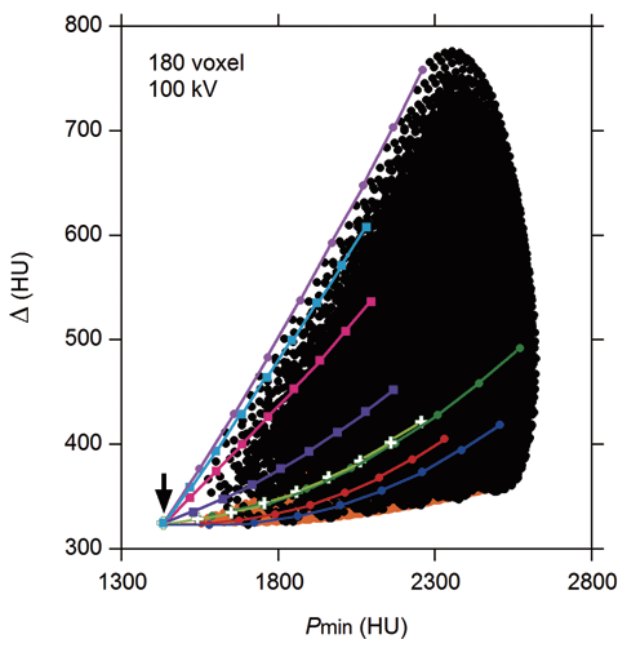

(c)
- Mixture of ten heavy elements
- Gd only
क Hf only
- Au only

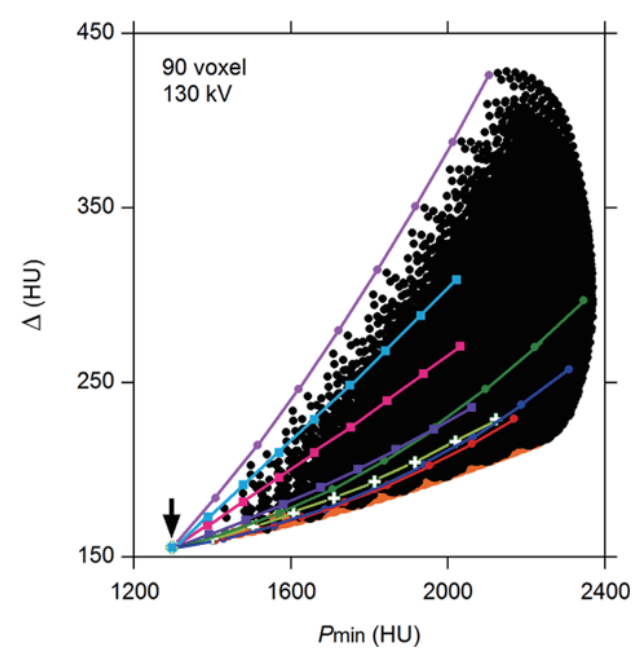

(b)

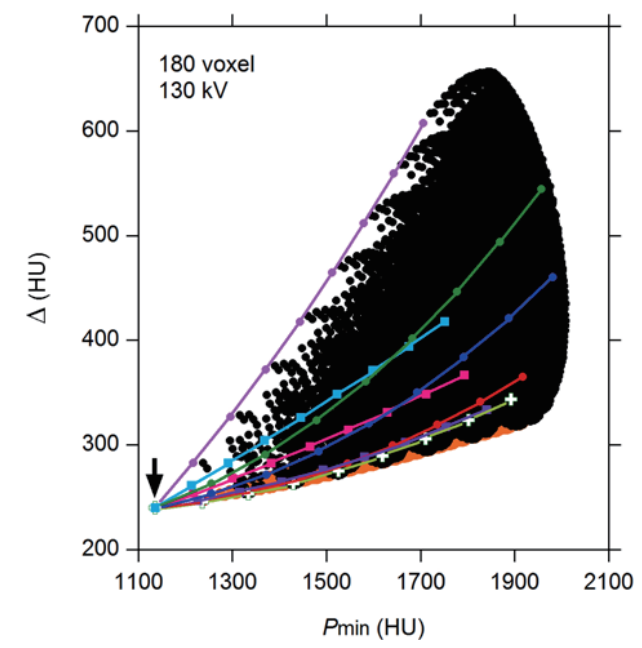

(d)

Fig. 4. Dependence of $P_{\min }$ and $\Delta$ on the chemical composition of the contrast agent. Sand-pack diameter and acceleration voltage are indicated. "Mixture of ten heavy elements" refers to a mixture of I, Ce, Nd, Gd, Ho, Yb, Hf, W, Au, and Bi for (a)-(d); the explored concentration range was from 0 to $8 \mathrm{wt} . \%$ (in increments of $1 \mathrm{wt} . \%$ with the further constraint that the maximum total heavy-element concentration was $8 \mathrm{wt} . \%$ ). "Mixture of four heavy elements" refers to mixtures of Gd, Ho, $\mathrm{Yb}$, and $\mathrm{Hf}$ for (a), Ho, Yb, Hf, and $\mathrm{W}$ for (b) and (c), and $\mathrm{Yb}, \mathrm{Hf}, \mathrm{W}$, and $\mathrm{Au}$ for (d); the examined concentration range was from 0.25 to $8 \mathrm{wt} . \%$ (in increments of $0.25 \mathrm{wt} . \%$ with the same further constraint that the maximum total heavy-element concentration was $8 \mathrm{wt} . \%$ ). "I only" denotes that only iodine was used as a heavy element in the aqueous suspension (from 0 to $8 \mathrm{wt} . \%$ with an increment of $1 \mathrm{wt} . \%$ ). The porous sand packs were saturated with aqueous fluid having various chemical compositions mentioned above. Various CT images of the fluid-filled sand packs were synthesized, and the line-profile analysis was applied to the images to obtain $P_{\min }$ and $\Delta$ as plotted in this figure. The result for the sand pack saturated with contrast agent-free pure water is indicated by a downward arrow in the bottom-left of the figures. 
Table 1

Chemistry in wt.\% of the eight best mixtures of contrast agents (A1 to D2 in Fig. 5). LAC spectra of sand-pack samples saturated with A2 or D2 are shown in Fig. 1, and the corresponding line profiles are shown in Fig. 3. A CT image of a sand pack saturated with D2 is shown in Fig. 2a

\begin{tabular}{cllllllll}
\hline Symbol in Fig. 5 & ${ }_{64} \mathrm{Gd}$ & ${ }_{67} \mathrm{Ho}$ & ${ }_{70} \mathrm{Yb}$ & ${ }_{22} \mathrm{Hf}$ & ${ }_{74} \mathrm{~W}$ & ${ }_{79} \mathrm{Au}$ & $\mathrm{H}_{2} \mathrm{O}$ & Total \\
\hline A1 & 1.5 & 1.25 & 0.5 & 0.25 & & & 96.5 & 100 \\
A2 & 2.25 & 3 & 1 & 0.25 & & & 93.5 & 100 \\
B1 & & 1.75 & 0.5 & 1 & 0.25 & & 96.5 & 100 \\
B2 & & 2.25 & 2 & 2 & 0.25 & & 93.5 & 100 \\
C1 & & 2 & 1 & 0.25 & 0.25 & & 96.5 & 100 \\
C2 & & 2.5 & 2.25 & 0.75 & 0.25 & & 94.25 & 100 \\
D1 & & 1 & 1.5 & 1.75 & 0.25 & 95.5 & 100 \\
D2 & & 1.5 & 2 & 2.5 & 1.5 & 92.5 & 100 \\
\hline
\end{tabular}

suspension was $8 \mathrm{wt} . \%$. The number of data points (orange circles) representing the mixtures of four heavy elements totals 35,960 in each figure of Figs 4 and 5. The concentration was increased in 0.25 wt.\% increments, which provided four times the resolution of the simulation for the mixture of 10 heavy elements. This high-resolution exploration enabled the dense plotting of the orange data points in Fig. 5, which was essential for the accurate determination of the contrast agent with the best chemistry. Table 1 shows eight examples for the mixtures of four heavy elements that produced the smallest $\Delta$ value for a specified $P_{\min }$ value in Fig. 5.

We also examined three additional mixtures of series of ten $Z$-heavy elements: ${ }_{63} \mathrm{Eu}$ to ${ }_{72} \mathrm{Hf}$ (sandpack diameter, 90 voxels; acceleration voltage, $100 \mathrm{kV}$ ), ${ }_{66}$ Dy to ${ }_{75} \mathrm{Re}$ (sand-pack diameter, 90 voxels; acceleration voltage, $130 \mathrm{kV}$; or sand-pack diameter, 180 voxels; acceleration voltage, $100 \mathrm{kV}$ ), and ${ }_{70} \mathrm{Yb}$ to ${ }_{79} \mathrm{Au}$ (sand-pack diameter, 180 voxels; acceleration voltage, $130 \mathrm{kV}$ ). However, we found no significant improvement compared with the mixtures of four heavy elements. Therefore, the results for these additional mixtures of ten $Z$-heavy elements are not shown in Figs 4 and 5.

The $P_{\min }$ of the sand-pack saturated with pure water exceeds $1000 \mathrm{HU}$, which is clearly larger than that of bulk water $(\approx 0 \mathrm{HU})$ (Fig. 4a-d, arrows). This means that a contrast agent with a larger concentration is required compared with that used for the imaging of the soft tissues of humans and animals, which are typically much lower in density. However, high-concentration contrast agents induce marked cupping. The degree of cupping, $\Delta$, shows clear $Z$-dependence (see colored lines in Fig. 4). This is a consequence of (i) the suppression of beam hardening occurring due to the $\mathrm{K}$ absorption edge at which the X-ray absorption abruptly increases and (ii) the energy of the $\mathrm{K}$ absorption edge being strongly $Z$-dependent [10]. As a result, each heavy element shows a unique trajectory in Figs 4 and 5.

\section{Discussion}

The interpretation of Figs 4 and 5 is shown in Fig. 6. The numerous black and orange data points in Figs 4 and 5 fall within a triangle-shaped grey region in Fig. 6. One of the three edges of the triangle indicated by a broken line (i.e., line AC) represents the worst fluid chemistry because it yields the largest $\Delta$ value, $\Delta_{2}$, for a specified $P_{\min }$ value, $P_{1}$; another edge, solid line $\mathrm{AB}$, denotes the best one because it gives the smallest $\Delta$ value, $\Delta_{1}$, for the specified $P_{1}$ value.

In two-phase Darcy flow laboratory experiments [3,4,15,27], porous media saturated with contrast agent-free pure water (e.g., case A in Fig. 6) are contiguously injected with fluid having a contrast agent, and the time-dependent degree of the local fluid replacement is monitored by the partial-volume effect [13-15] in CT images. If $\Delta_{0}=\Delta_{1}$ in Fig. 6(b), the cupping effect is derived only from the sand 
- Mixture of four heavy elements
- I only
- Ho only

- $\mathrm{Yb}$ only

- W only

- Bi only

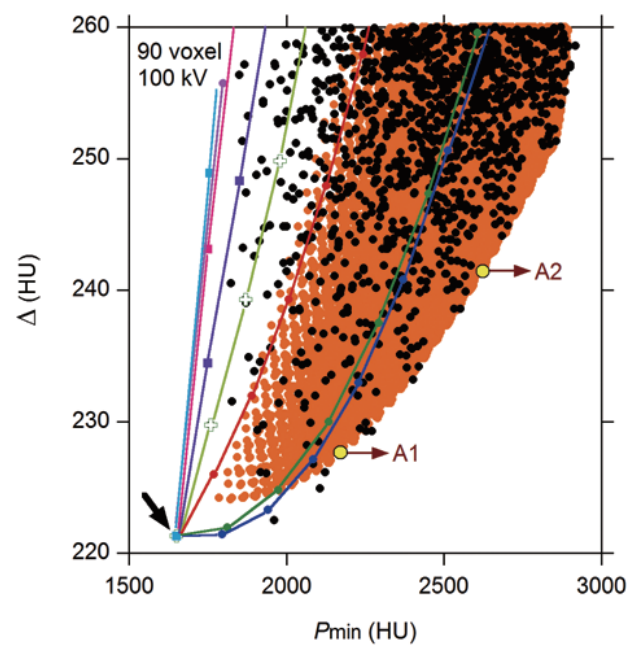

(a)

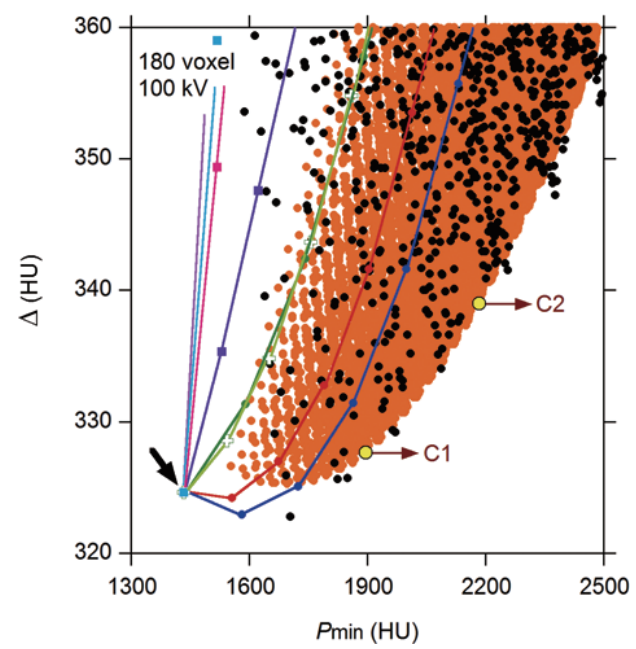

(c)
- Mixture of ten heavy elements $\mathrm{Hf}$ only

- Gd only

- Au only

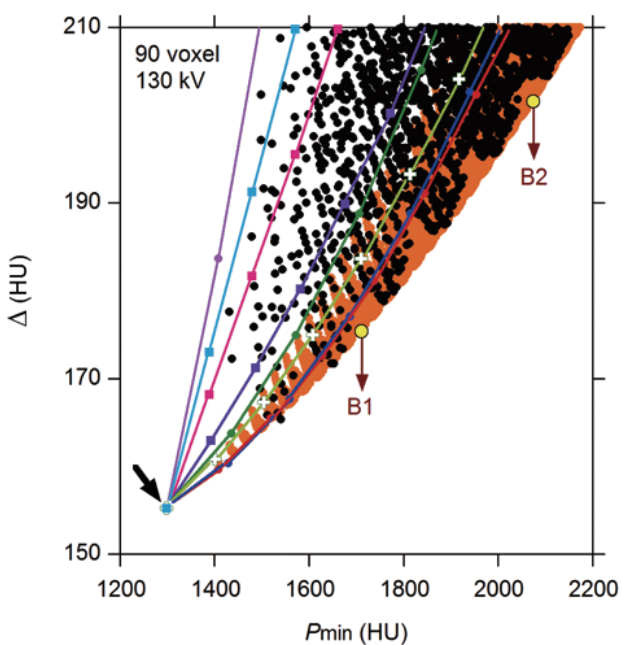

(b)

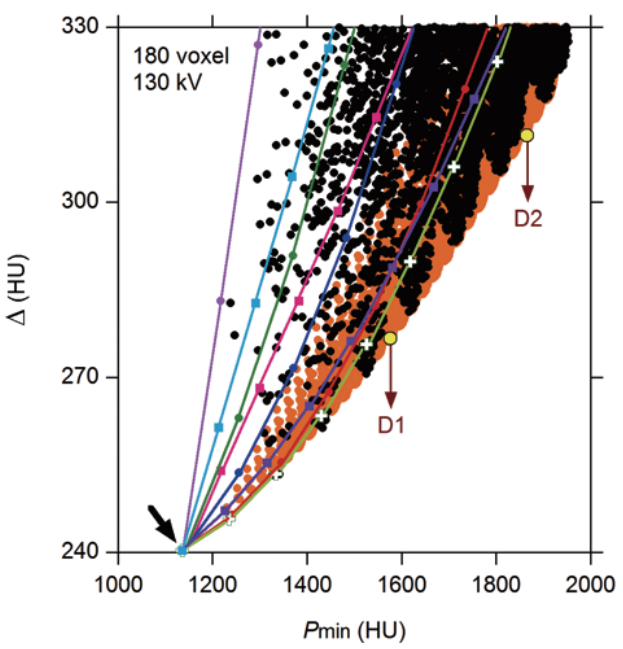

(d)

Fig. 5. Enlargement of Fig. 4. Eight examples of the best contrast agents yielding the smallest $\Delta$ value for a fixed $P_{\text {min }}$ are indicated as yellow data points with the letters A1 to D2 in (a)-(d). The chemical compositions of these contrast agents are listed in Table 1.

grains and the contrast agent superimposes no cupping on the CT images. This pre-existing cupping derived from the sand grains before the fluid replacement can be completely canceled by taking it as a reference image during the image subtraction processing [27]. Thus, the case $\Delta_{0}=\Delta_{1}$ is ideal because no cupping-derived error occurs in the calculation of the degree of the local fluid replacement. Unfortunately the simulation results show that $\Delta_{1}$ is slightly larger than $\Delta_{0}$ in Fig. 5 , and the best line $\mathrm{AB}$ is not horizontal but inclined in Fig. 6(a). As a result, a certain degree of error derived from the slight difference of $\Delta_{1}$ and $\Delta_{0}$ inevitably occurs even for the best case in Fig. 6. However, Fig. 5 shows that 


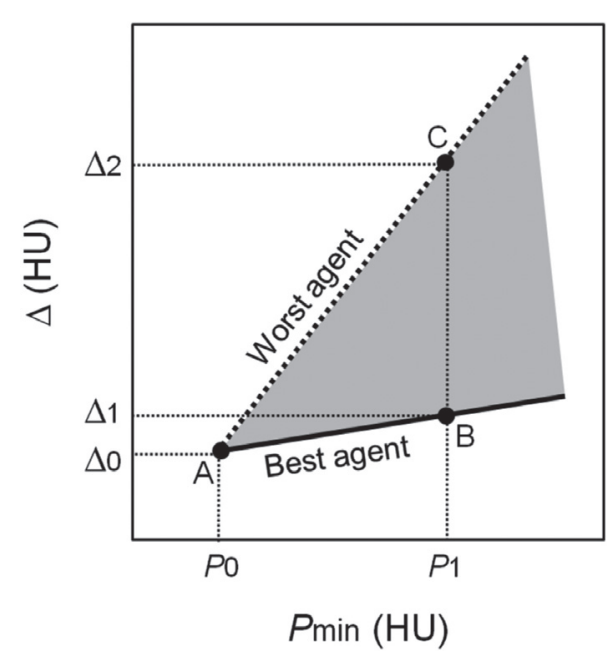

(a)

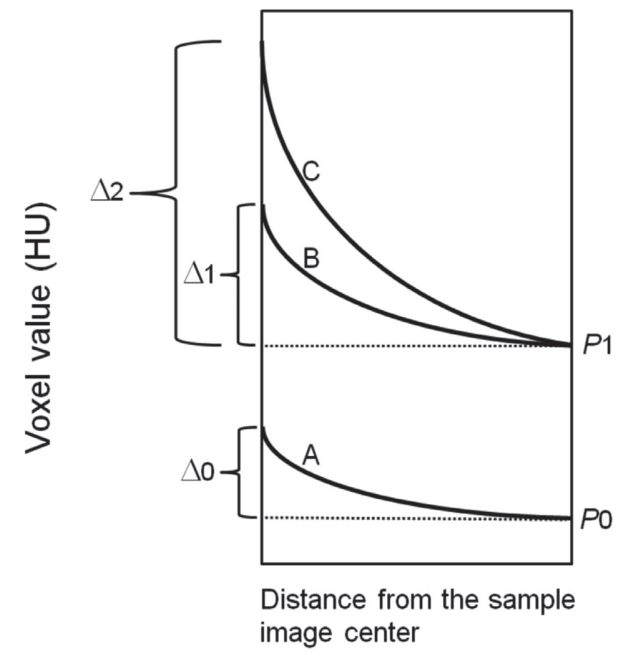

(b)

Fig. 6. Interpretation of Figs 4 and 5. (a) Schematics of Fig. 5. The case A denotes the simulation result for the sand pack saturated with contrast agent-free pure water. (b) Corresponding line profiles along the baselines for the three cases in (a).

$\Delta_{1}-\Delta_{0}<<\Delta_{2}-\Delta_{0}$, suggesting that the use of the best contrast agent greatly suppresses the cupping artifact derived from the contrast agent compared with that of the worst contrast agent. Thus, it is meaningful to choose the best contrast agent for the CT image-based calculations of the relative permeability curve and residual oil saturation [12] by means of the partial-volume effect in two-phase Darcy flow experiments $[3,4,15]$.

The use of iodine as a single heavy element corresponds to the worst case in Fig. 6(a) because it yields very large $\Delta$ values compared with the other heavy elements (see purple lines with circles in Fig. 4(a)(d)). This is a consequence of the $\mathrm{K}$ absorption edge of iodine being located at $33.2 \mathrm{keV}$, which is far from the peak of the X-ray source spectrum (Fig. 1), and the beam hardening reduction by the abrupt increase of the LAC at the $\mathrm{K}$ absorption edge does not work effectively. Iodine was not included in the six heavy elements listed in Table 1, indicating that mixing of iodine with other heavy elements does not contribute to the beam hardening reduction. Thus, we do not recommend iodine as a contrast agent for geotechnical CT experiments using high-density samples.

Figures 3 and 4 show that aqueous suspensions of a single element much heavier than ${ }_{53} \mathrm{I}$ induce markedly less cupping. The use of elements ranging from ${ }_{64} \mathrm{Gd}$ to ${ }_{79} \mathrm{Au}$ is recommended (Figs 4 and 5); for example, in Fig. 4(c), an aqueous suspension of ${ }_{67} \mathrm{Ho}$ (blue line with circles) produces about half the cupping compared with an aqueous suspension of iodine (purple line with circles) for the same $P_{\text {min }}$ value. It should be noted that ${ }_{64} \mathrm{Gd}$, which is recommended for the imaging of the low-density soft tissues of humans [25,26], is not always the most suitable contrast agent for high-density sand-pack imaging because elements heavier than gadolinium generally yield even smaller $\Delta$ values (Fig. 4).

Nakashima [27] performed a CT imaging experiment by using a Toyoura sand-pack (diameter, $56 \mathrm{~mm}$; porosity, $39 \mathrm{vol} . \%$; acceleration voltage, $130 \mathrm{kV}$ ), and found that an aqueous solution of a tungstenbearing electrolyte $\left(\mathrm{Na}_{6} \mathrm{H}_{2} \mathrm{~W}_{12} \mathrm{O}_{40}\right)$ markedly reduced cupping compared with a KI solution, showing that tungsten is a potentially useful contrast agent in hydrological CT experiments. This finding is confirmed in Figs 3 and $4 \mathrm{~d}$ where an aqueous suspension of tungsten yielded much smaller $\Delta$ values compared with iodine. 
The best contrast agents in Fig. 6(a) are the mixtures of four heavy elements from ${ }_{64} \mathrm{Gd}$ to ${ }_{79} \mathrm{Au}$ because the mixtures yield the smallest $\Delta$ value for a specified $P_{\min }$ value throughout most of the conditions examined (Figs 4 and 5). The LAC spectra in Fig. 1 for the optimized contrast agents listed in Table 1 have flatter, less energy-dependent distributions compared with that of iodine due to the combined increase in LAC at the four $\mathrm{K}$ absorption edges. Flatter LAC spectra yield less cupping (Figs 2a and 3). As the X-ray passes through the sample or the acceleration voltage increases, the X-ray spectrum becomes hard [6], resulting in the rightward shift of the spectrum peak in Fig. 1. Thus, a heavier element with a $\mathrm{K}$ absorption edge occurring at a larger energy is required to effectively suppress beam hardening. As a result, the atomic number of the most suitable four heavy elements increases with acceleration voltage and sample diameter (Table 1). Less toxic and chemically stable compounds containing lanthanoids, hafnium, tungsten, or gold have previously been used as contrast agents in the medical and life sciences [29,31-35]. Thus, it is straightforward to mix the compounds to prepare the contrast agents listed in Table 1.

In relation to the current study, the following points should be noted:

1. The best contrast agent depends on the acceleration voltage and sample size (Figs 4 and 5). Although the results are not shown, we performed supplementary CT simulations showing that the best contrast agent depends also on the chemical composition and porosity of the geo-material being imaged. Therefore, further CT image simulations should be performed to find the best contrast agent for the size, chemical composition, and porosity of the sample being examined, and the acceleration voltage being used.

2. When using bare heavy cations such as $\mathrm{Yb}^{3+}$ as contrast agents, the ions may be immobilized on the negatively charged mineral surfaces of geological samples such as clays [39]. The use of a chelate complex [34] is a possible solution to this problem.

3. The present study is focused on the reduction of the cupping effect inside homogeneous samples. Another artifact derived from beam hardening can occur for the hydrological CT of the preferential flow in macroscopic pipes/fractures inside heterogeneous porous samples; it is a dark streak artifact which arises between two pipes/fractures having bright voxel values. The dark streak artifact also reduces the accuracy of post-CT outcomes such as image-based calculation of the degree of the local fluid replacement using the partial-volume effect. Fortunately, previous studies $[9,26]$ reported that the reduction of the cupping artifact also yields the reduction of the dark streaks. Thus, the outcome of the present study for the reduction of cupping would also contribute to the reduction of the dark streak artifact critical for the hydrological CT experiments of the preferential flow in macroscopic pipes and fractures.

4. Our method presented here does not exclude the simultaneous use of other conventional techniques to reduce beam hardening. For example, if the increase in random noise that occurs when the intensity of the X-ray is decreased is allowable in terms of the research being undertaken, doing so, together with using a metal filter [20,21], is a possible solution for further reducing beam hardening artifacts.

\section{Conclusions}

To facilitate the nonmedical application of polychromatic X-ray CT systems to the quantitative analysis of high-density samples, computer simulations were performed to optimize the chemistry of contrast agents to induce the least cupping in a fluid-saturated porous sand-pack image. Iodine, the most commonly used contrast agent in earth sciences, is not recommended because it induces marked cupping. 
This is a result of the $\mathrm{K}$ absorption edge of iodine being far from the peak of the X-ray source spectrum. A single element heavier than iodine, ranging from gadolinium to gold, induced less cupping artifact. A carefully optimized mixture of four heavy elements chosen from gadolinium to gold induced the least cupping.

\section{Acknowledgements}

Comments by the anonymous reviewers were helpful. $\mathrm{R}$ language programming was performed by S. Kamiya. The public domain program, ImageJ, developed by the National Institutes of Health and available at http://imagej.nih.gov/ij/ was used for image processing (e.g., line profile analysis) of the simulated CT images. Preliminary CT experiments of Toyoura sand pack samples were performed under the cooperative research program of Center for Advanced Marine Core Research (CMCR), Kochi University (13B034) with the support of JAMSTEC.

\section{References}

[1] H. Lusic and M.W. Grinstaff, X-ray-computed tomography contrast agents, Chem Rev 113 (2013), 1641-1666.

[2] H.M.D. Agbogun, T.A. Al and E.M.A. Hussein, Three dimensional imaging of porosity and tracer concentration distributions in a dolostone sample during diffusion experiments using X-ray micro-CT, J Contam Hydrol 145 (2013), 44-53.

[3] T.J. Heindel, A review of X-ray flow visualization with applications to multiphase flows, J Fluids Eng 133 (2011), article number 074001

[4] S. Uemura, D. Fukabori, S. Tsushima, and S. Hirai, Visualization and analysis of $\mathrm{CO}_{2}$ permeation process in a porous media by microfocus X-ray computed tomography, Trans Jpn Soc Mech Eng Ser B 78 (2012), 74-82.

[5] O.P. Wennberg, L. Rennan, and R. Basquet, Computed tomography scan imaging of natural open fractures in a porous rock; geometry and fluid flow, Geophys Prospect 57 (2009), 239-249.

[6] J.F. Barrett and N. Keat, Artifacts in CT: Recognition and avoidance. Radiographics 24 (2004), 1679-1691.

[7] R.A. Brooks and G. Di Chiro, Beam hardening in x-ray reconstructive tomography, Phys Med Biol 21 (1976), 390-398.

[8] Z. Jovanović, F. Khan, F. Enzmann, and M. Kersten, Simultaneous segmentation and beam-hardening correction in computed microtomography of rock cores, Comp Geosci 56 (2013), 142-150.

[9] K. Remeysen and R. Swennen, Beam hardening artifact reduction in microfocus computed tomography for improved quantitative coal characterization, Int J Coal Geol 67 (2006), 101-111.

[10] Y. Nakashima and T. Nakano, Nondestructive quantitative analysis of a heavy element in solution or suspension by single-shot computed tomography with a polychromatic X-ray source, Anal Sci 28 (2012), 1133-1138.

[11] M.D. Wong, X. Wu and H. Liu, The effects of X-ray beam hardening on detective quantum efficiency and radiation dose, J X-Ray Sci Technol 19 (2011), 509-519.

[12] E.C. Donaldson, G.V. Chilingarian and T.F. Yen, eds, Enhanced Oil Recovery, I: Fundamentals and Analyses, Elsevier, Amsterdam, 1985.

[13] A. Guvenilir, T.M. Breunig, J.H. Kinney and S.R. Stock, New direct observations of crack closure processes in Al-Li 2090 T8E41, Phil Trans R Soc London A 357 (1999), 2755-2775.

[14] A. Guvenilir, T.M. Breunig, J.H. Kinney and S.R. Stock, Direct observation of crack opening as a function of applied load in the interior of a notched tensile sample of Al-Li 2090, Acta Mater 45 (1997), 1977-1987.

[15] S.C.M. Krevor, R. Pini, L. Zuo and S.M. Benson, Relative permeability and trapping of $\mathrm{CO}_{2}$ and water in sandstone rocks at reservoir conditions, Water Resour Res 48 (2012), article number W02532.

[16] L. Brabant, E. Pauwels, M. Dierick, D. Van Loo, M.A. Boone and L. Van Hoorebeke, A novel beam hardening correction method requiring no prior knowledge, incorporated in an iterative reconstruction algorithm, NDT and E Int 51 (2012), 68-73.

[17] M. Krumm, S. Kasperl and M. Franz, Reducing non-linear artifacts of multi-material objects in industrial 3D computed tomography, NDT and E Int 41 (2008), 242-251.

[18] T. Nakano, Y. Nakashima, K. Nakamura, and S. Ikeda, Observation and analysis of internal structure of rock using X-ray CT, J Geol Soc Jpn 106 (2000), 363-378.

[19] S. Tang, X. Mou, Y. Yang, Q. Xu, and H. Yu, Application of projection simulation based on physical imaging model to the evaluation of beam hardening corrections in X-ray transmission tomography, J X-Ray Sci Technol 16 (2008), 95-117. 
[20] J.A. Meganck, K.M. Kozloff, M.M. Thornton, S.M. Broski and S.A. Goldstein, Beam hardening artifacts in microcomputed tomography scanning can be reduced by X-ray beam filtration and the resulting images can be used to accurately measure BMD, Bone 45 (2009), 1104-1116.

[21] M. Van Geet, R. Swennen and M. Wevers, Quantitative analysis of reservoir rocks by microfocus X-ray computerised tomography, Sed Geol 132 (2000), 25-36.

[22] A.J. Coleman and M. Sinclair, A beam-hardening correction using dual-energy computed tomography, Phys Med Biol 30 (1985), 1251-1256.

[23] H. Long, R. Swennen, A. Foubert, M. Dierick and P. Jacobs, 3D quantification of mineral components and porosity distribution in Westphalian C sandstone by microfocus X-ray computed tomography, Sed Geol 220 (2009), 116-125.

[24] A. Tsuchiyama, T. Hanamoto, Y. Nakashima and T. Nakano, Quantitative evaluation of attenuation contrast of minerals by using a medical X-ray CT scanner, J Miner Petrol Sci 95 (2000), 125-137.

[25] H.N. Cardinal, D.W. Holdsworth, M. Drangova, B.B. Hobbs and A. Fenster, Experimental and theoretical x-ray imaging performance comparison of iodine and lanthanide contrast agents, Med Phys 20 (1993), 15-31.

[26] C. Ruth and P.M. Joseph, A comparison of beam-hardening artifacts in X-ray computerized tomography with gadolinium and iodine contrast agents, Med Phys 22 (1995), 1977-1982.

[27] Y. Nakashima, The use of sodium polytungstate as an X-ray contrast agent to reduce the beam hardening artifact in hydrogeological laboratory experiments, J Hydrol Hydromech 61 (2013), 347-351.

[28] J. Katagiri, T. Matsushima and Y. Yamada, Simple shear simulation of 3D irregularly-shaped particles by image-based DEM, Granul Matter 12 (2010), 491-497.

[29] J.F. Hainfeld, D.N. Slatkin, T.M. Focella and H.M. Smilowitz, Gold nanoparticles: a new X-ray contrast agent, Brit J Radiol 79 (2006), 248-253.

[30] O. Rabin, J.M. Perez, J. Grimm, G. Wojtkiewicz and R. Weissleder, An X-ray computed tomography imaging agent based on long-circulating bismuth sulphide nanoparticles, Nature Materials 5 (2006), 118-122.

[31] T. Albrecht and P. Dawson, Gadolinium-DTPA as X-ray contrast medium in clinical studies, Brit J Radiol 73 (2000), $878-882$.

[32] K.E. deKrafft, W.S. Boyle, L.M. Burk, O.Z. Zhou, and W. Lin, Zr-and Hf-based nanoscale metal-organic frameworks as contrast agents for computed tomography, J Mater Chem 22 (2012), 18139-18144.

[33] Y. Liu, K. Ai, J. Liu, Q. Yuan, Y. He and L. Lu, A high-performance ytterbium-based nanoparticulate contrast agent for in vivo X-ray computed tomography imaging, Angew Chem Int Ed 51 (2012), 1437-1442.

[34] H. Pietsch, G. Jost, T. Frenzel, M. Raschke, J. Walter, H. Schirmer, J. Hütter and M.A. Sieber, Efficacy and safety of lanthanoids as X-ray contrast agents, Eur J Radiol 80 (2011), 349-356.

[35] S.R. Stock, S. Nagaraja, J. Barss, T. Dahl and A. Veis, X-ray microCT study of pyramids of the sea urchin Lytechinus variegatus, J Struct Biol 141 (2003), 9-21.

[36] M.J. Berger, J.H. Hubbell, S.M. Seltzer, J. Chang, J.S. Coursey, R. Sukumar, D.S. Zucker and K. Olsen, XCOM: Photon Cross Sections Database (National Institute of Standards and Technology), http://www.nist.gov/pml/data/xcom/index. $\mathrm{cfm} /$.

[37] W.H. Haynes, ed., CRC Handbook of Chemistry and Physics, CRC Press, London, 2010.

[38] Y. Nakashima, $\mathrm{H}_{2} \mathrm{O}$ self-diffusion coefficient of water-rich MX-80 bentonite gels, Clay Miner 41 (2006), 659-668.

[39] Y. Nakashima, Diffusivity measurement of heavy ions in Wyoming montmorillonite gels by X-ray computed tomography, J Contam Hydrol 61 (2003), 147-156. 